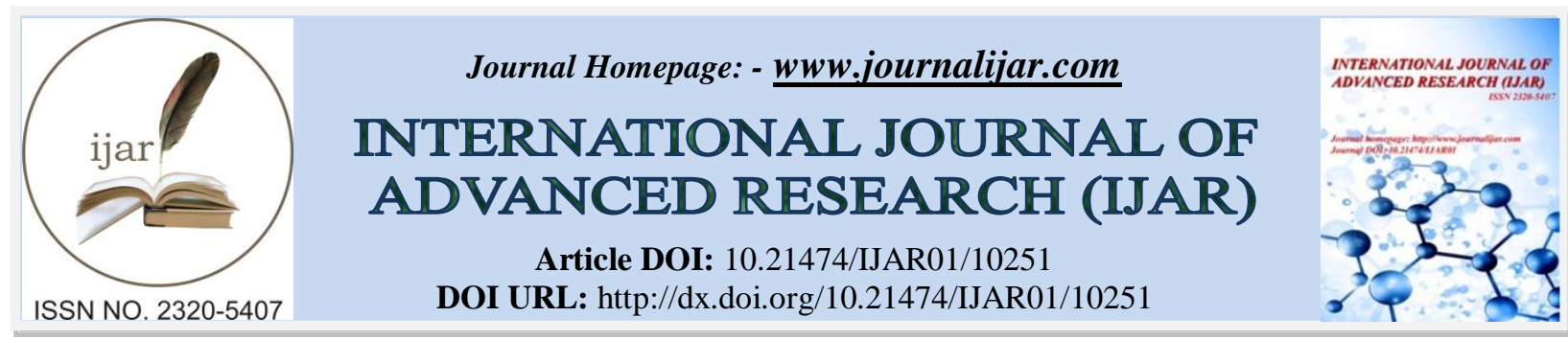

RESEARCH ARTICLE

\title{
FIBROELASTOMA OF THE TRICUSPID VALVE ASSOCIATED WITH HEAVY CARDIAC SURGERY : A CASE REPORT
}

\section{Dr. Lamdak Soufiane ${ }^{1}$ and Dr. Raoui Jihad ${ }^{2}$}

1. Resident in Cardiology at Ibn sina Rabat University Hospital, Morroco, Intern at the Cardiology Department at Henri Mondor Hospital in Aurillac.

2. Resident in Cardiology at Ibn sina Rabat University Hospital, Morroco.

\section{Manuscript Info}

\section{Manuscript History}

Received: 14 October 2019

Final Accepted: 16 November 2019

Published: December 2019

\section{Abstract}

Cardiac papillary fibroelastomas (EF) are rare cardiac tumors, but represent the most common of the valvular tumors. The majority of EFs is asymptomatic. However, EF of the left heart valves is more often associated with serious symptoms, Papillary fibroelastoma is a rare cause of embolic stroke. It is most frequently located in the aortic and mitral valves. The onset of symptoms and tumors that are mobile or larger than $10 \mathrm{~mm}$ are a surgical indication. Complete surgical resection of the tumor is curative, with no recurrence of long-term lesions.

Copy Right, IJAR, 2019,. All rights reserved.

\section{Introduction:-}

Cardiac papillary fibroelastomas (EF) are rare cardiac tumors, but represent the most common of the valvular tumors. The majority of EFs is asymptomatic. However, EF of the left heart valves is more often associated with serious symptoms. The EFs of the tricuspid valve, on the other hand, are often asymptomatic and accidentally discovered during an echocardiography, during a cardiac surgery intervention or during an autopsy. We report the case of EF of the tricuspid valve of incidental discovery of a patient who has syncopal discomfort associated with palpitations and dyspnea.

\section{Observation:-}

The patient O.M aged 71, with a history: HTA, smoking 40 packs / year, with a complex cardiological history; first hospitalization in July 2018 for discomfort in the context of hypotension and bradycardia at 40 / min. rhythmic monitoring found a few episodes of sinus dysfunction. the indication for a dual-chamber pacemaker was initially retained. Note the completion of a myocardial scintigraphy finding a sequel of infra-basal necrosis of 2 segments out of 17 , with a globally preserved LVEF.

She returned in the meantime to our services for a deterioration in the general condition, a crescendo dyspnea in a context of palpitation / tachycardia. The ECG in the emergency department highlights an atrial fibrillation at 140 / min followed by a spontaneous reduction with significant post reduction breaks. It is transferred to our intensive care unit cardiology for rhythmic monitoring. The clinical examination on admission found: a Weight: $46 \mathrm{~kg}$, height: 1.56 m, BMI: 18.43. Cardiovascular examination finds noises of the heart perceived at irregular rhythm, systolic Breath of mitral insufficiency side 4/6, No sign of right cardiac insufficiency. The pleuropulmonary examination finds some Crakes crackling at the two bases. The initial objective cardiac echocardiography: LV of limited size, 
slightly enlarged with preserved systolic function $(\mathrm{EF}=56 \%)$, seat of a large inferior basal / median aneurysmal pocket without endocavitary thrombus (Figure 1), altered, leaky mitral valve, significant mitral insufficiency of ischemic appearance (ROA: $0.41 \mathrm{~cm} 2, \mathrm{RV}: 45.77 \mathrm{ml}$ ) (Figure 5), altered aortic valve with significant aortic insufficiency (grade III) (Figure 6), a bulky mass appended to the atrial side of the tricuspid valve ( figure 2,3). The CRP at admission was 75 with a notion of febricule within 48 hours. The hypothesis of an infectious endocarditis on a native mitral valve was therefore advanced. TEE confirms the mass of the tricuspid valve (19*15 mm) (Figure 7), rounded, very mobile, no damage to the pulmonary valve. No argument for endocarditis of the aortic or mitral valve .1 the infero-basal aneurysm is perfectly visualized by the trans-gastric sections (Figure 4).

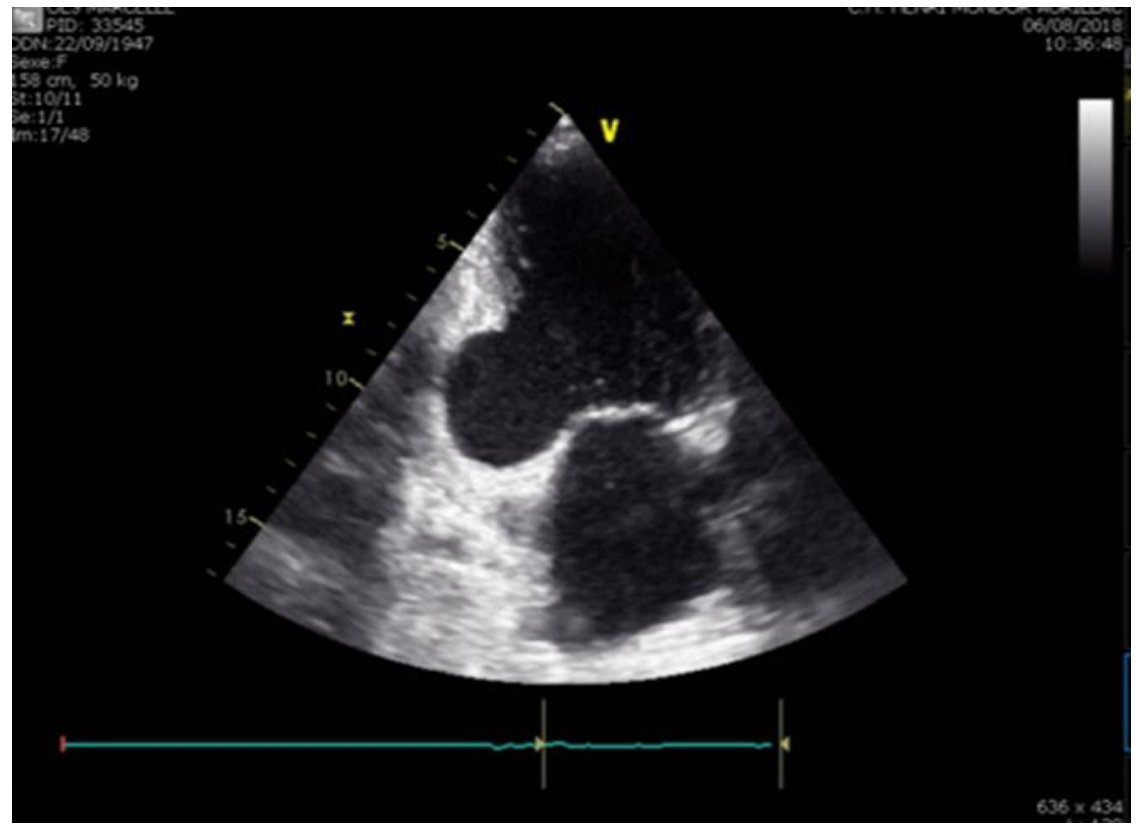

Figure 1:- Intercostal section of two cavities showing the inferobasal aneurysmal pocket.

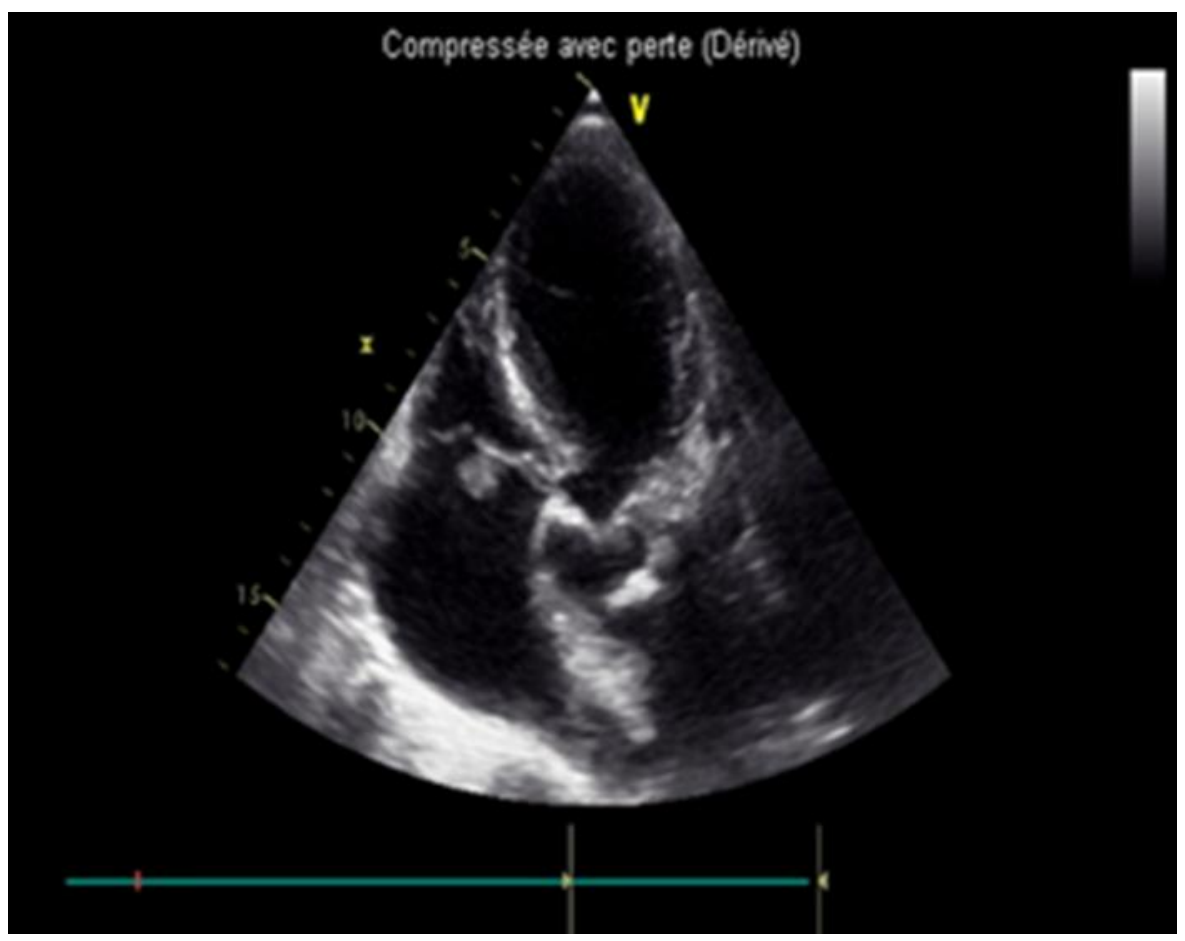

Figure 2:- Intersostal section with five cavities showing the tricuspid valve mass on the ear slope. 


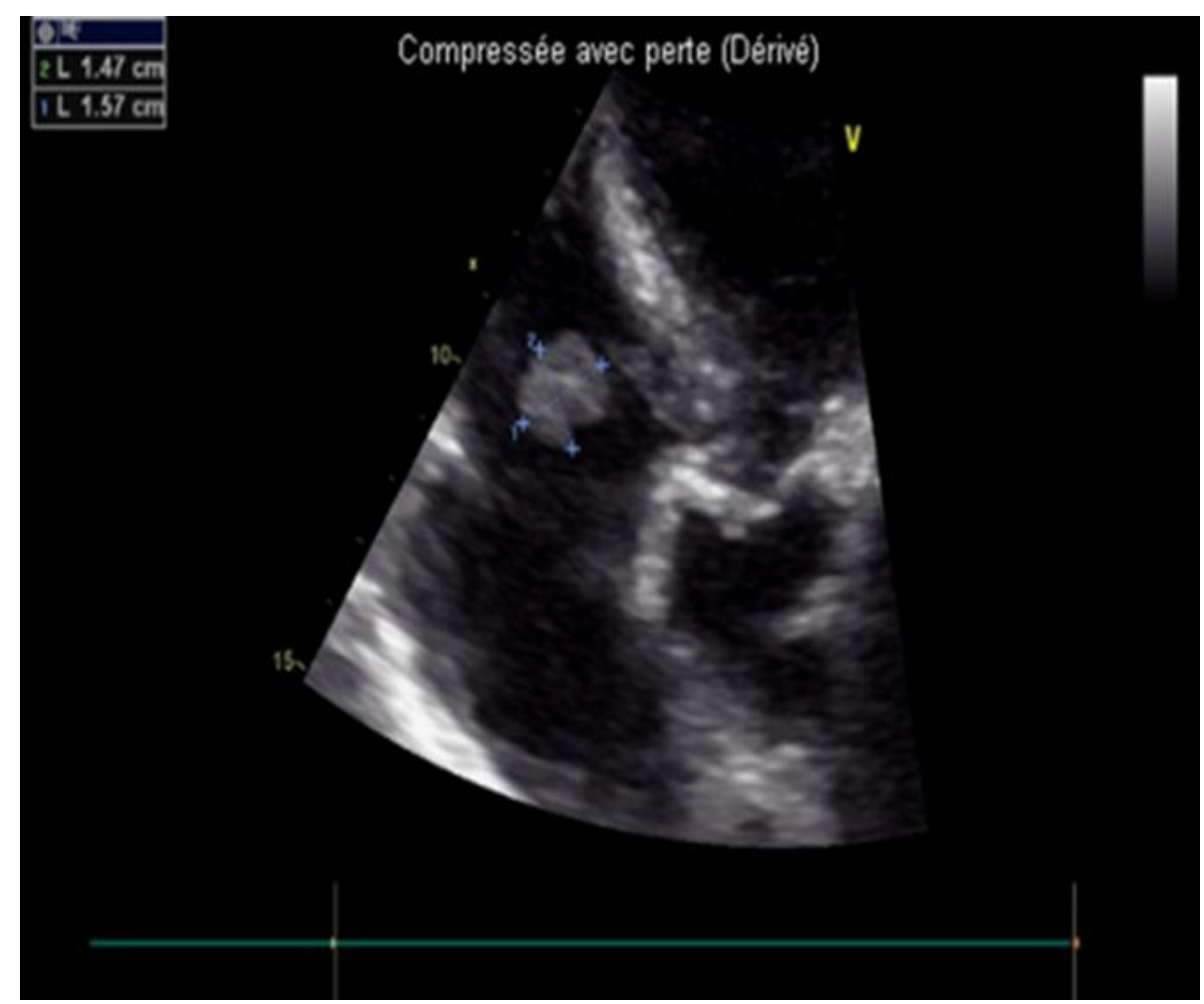

Figure 3:- five-chamber parasternal section showing the dimensions of the tricuspid valve mass $(15 * 16 \mathrm{~mm})$.

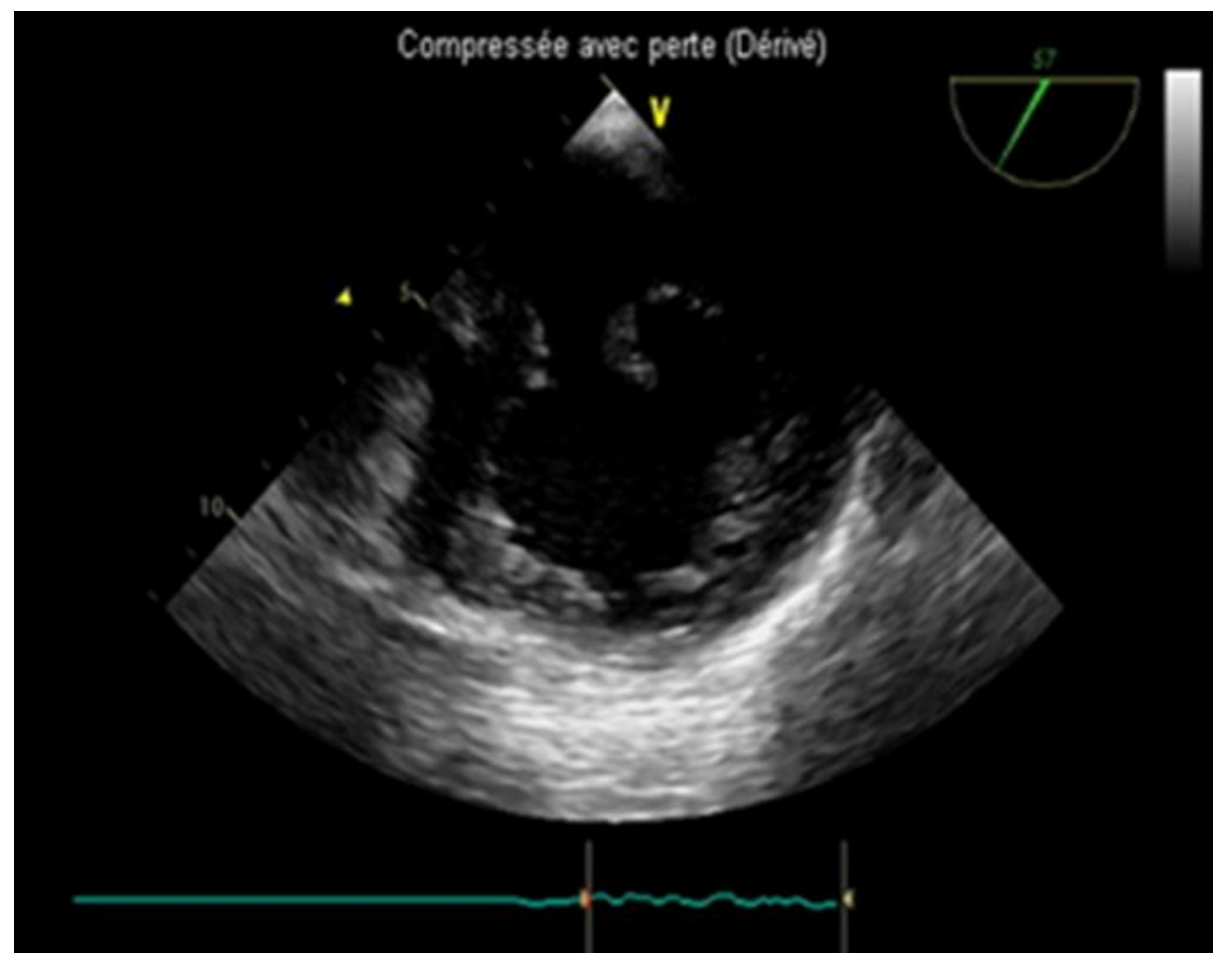

Figure 4:- TEE cross-sectional bidirectional short axis showing the aneurysmal pocket without endocavitary thrombus. 


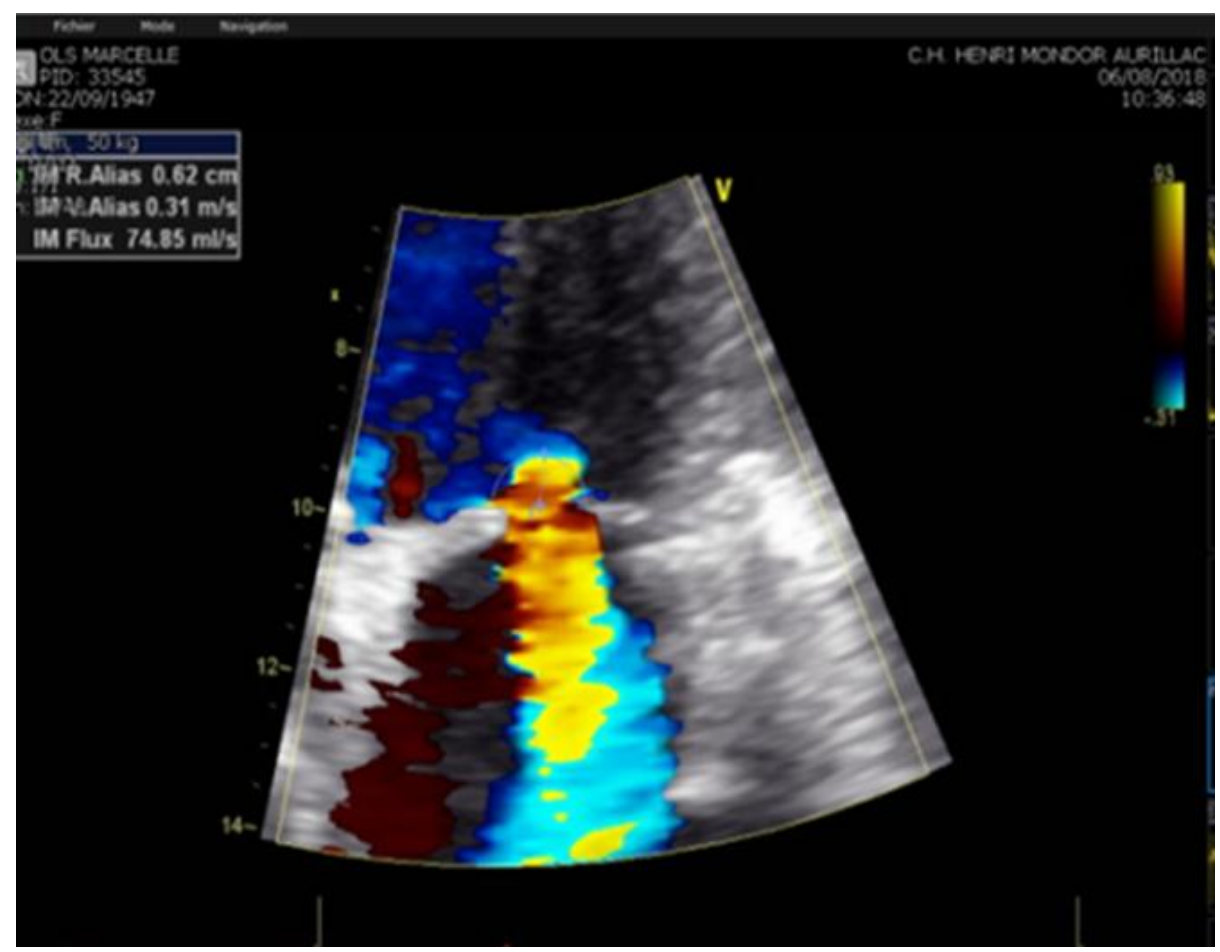

Figure 5:- Intercostal section 4 cavities with a zoom on the mitral valve objectifying the significant mitral insufficiency of ischemic appearance (ROA : $0.41 \mathrm{~cm} 2, \mathrm{RV}: 45.77 \mathrm{ml}$ ).

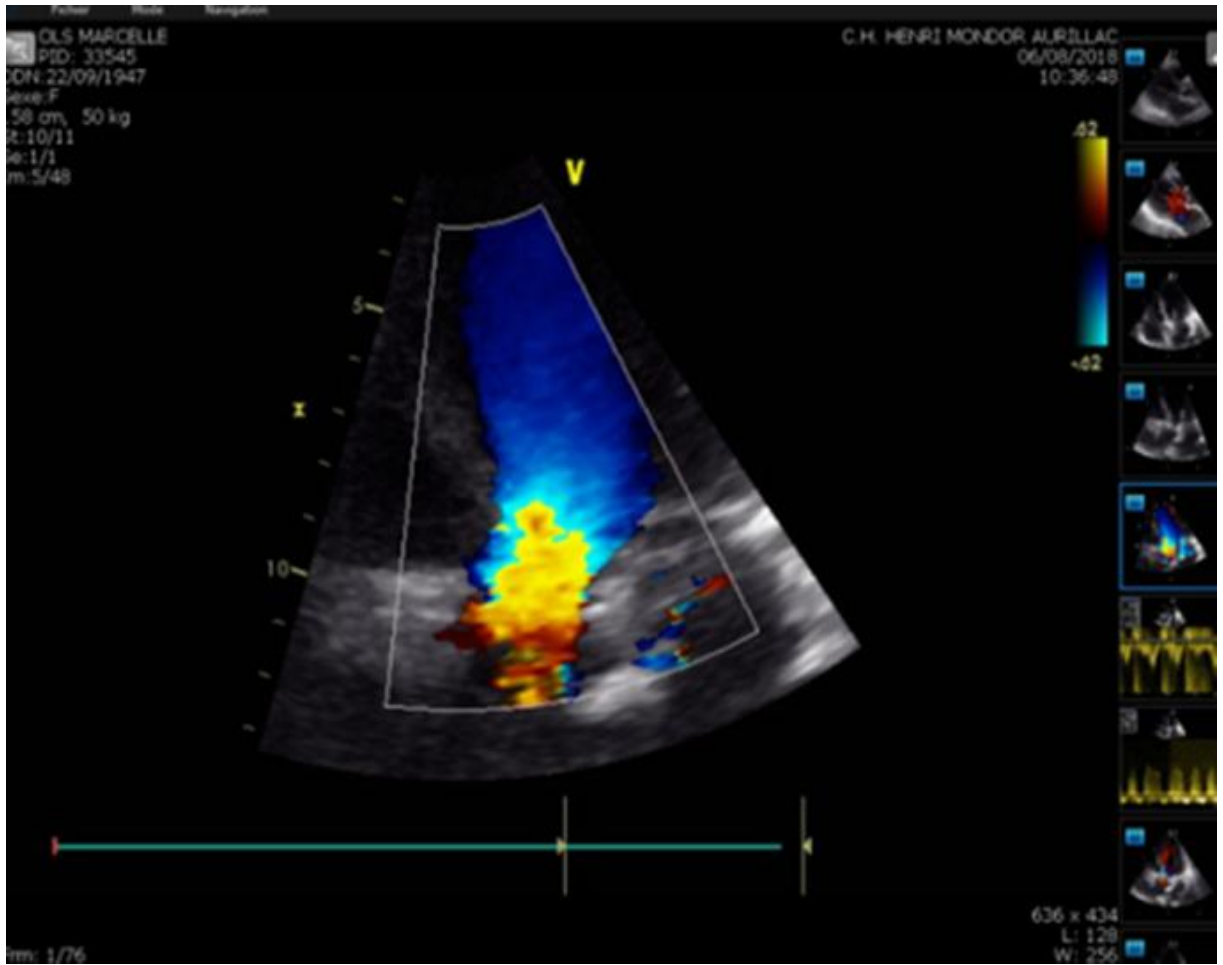

Figure 6:- Intercostal section of five cavities zooms in on the aortic valve objectifying the significant aortic leak. 


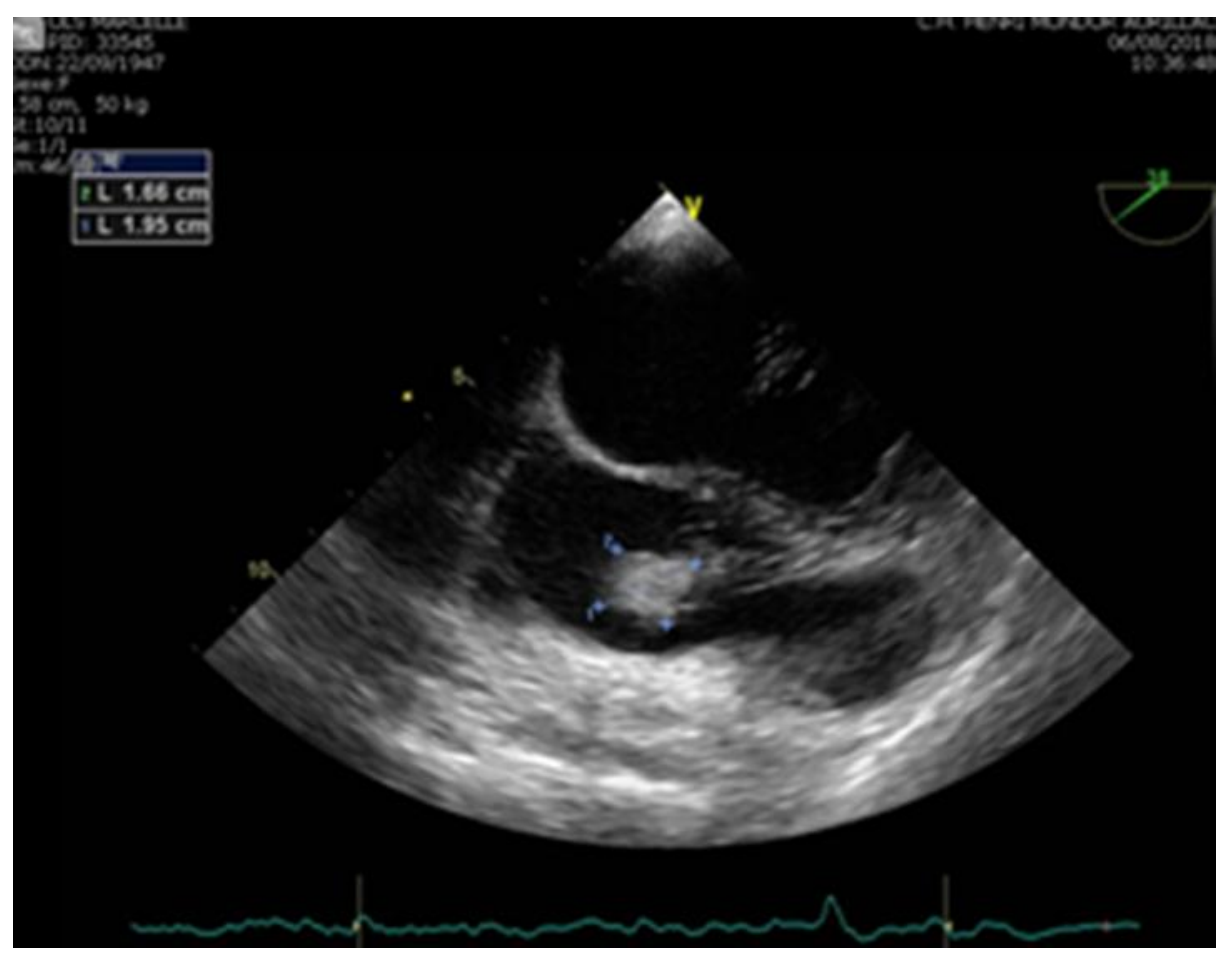

Figure 7:- Two-dimensional mid-oesophageal TEE section with four cavities showing the diameters of the tricuspid valve mass $(19 * 15 \mathrm{~mm})$.

No antibiotic therapy has been initiated pending the results of blood cultures. The entire infectious assessment (5 pairs of blood cultures) will come back sterile, just like the urine analysis, the culture being sufficiently long (endocarditis protocol) we can confirm the absence of active infection.

The patient benefited from a right coronary angiography, which objectified severe tri-trunk lesions with tight stenosis of the proximal right coronary followed by chronic occlusion of the middle segment weakly recovered (Figure 9). Tight stenosis of the common trunk, of the proximal anterior ventricle and the 1st diagonal (Figure 8). Tight stenosis of the proximal circumflex (Figure 8).

The ventriculography is impressive confirming the postero-basal aneurysm with moderate dilation of the left ventricle.

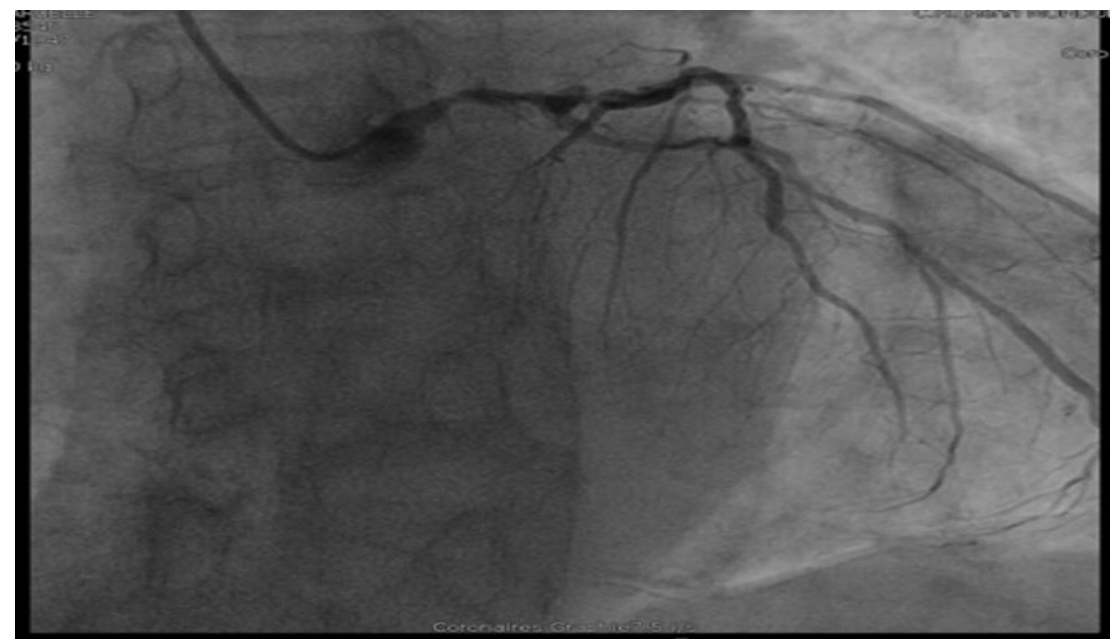

Figure 8:- Caudal incidence. showing a tight stenosis of the common trunk in its middle portion with a tight stenosis of the proximal circumflex artery. 


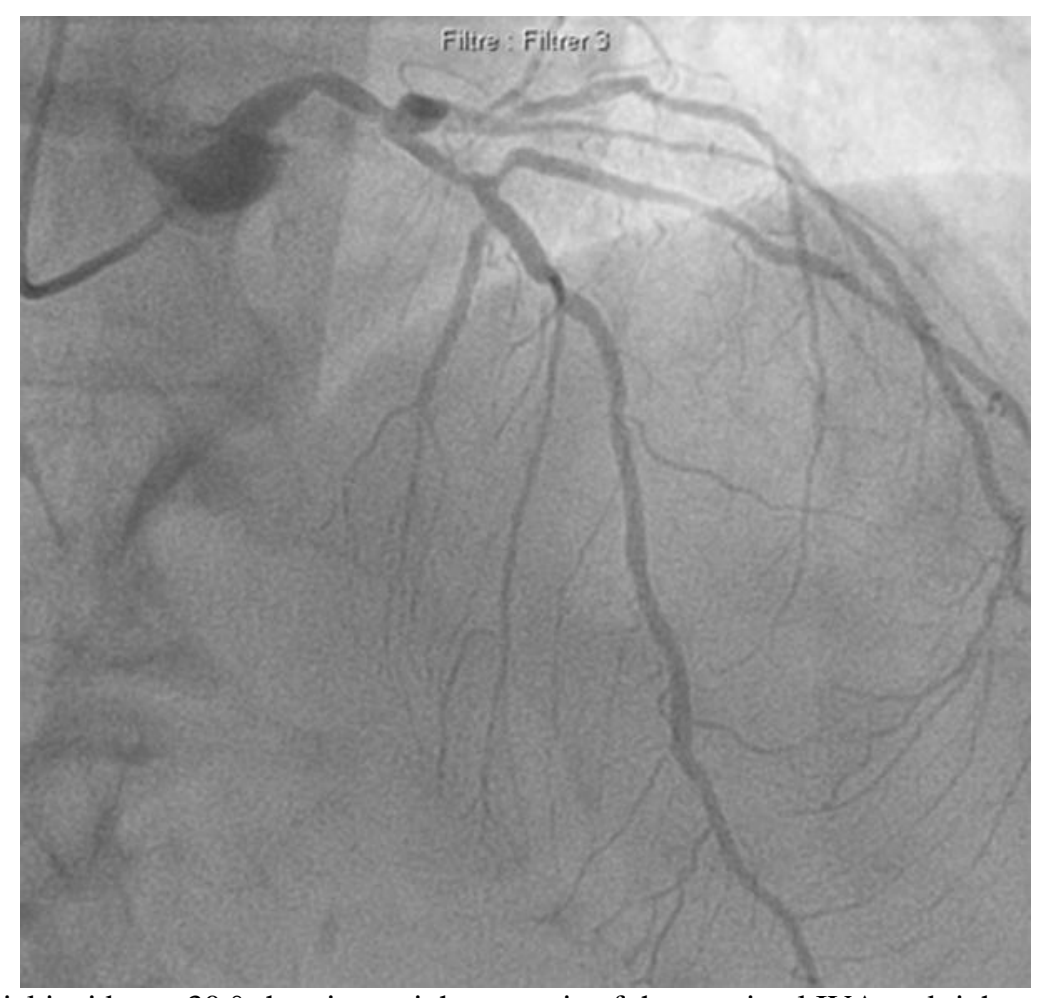

Figure 10:- cranial incidence $30^{\circ}$ showing a tight stenosis of the proximal IVA and tight stenosis of the first diagonal.

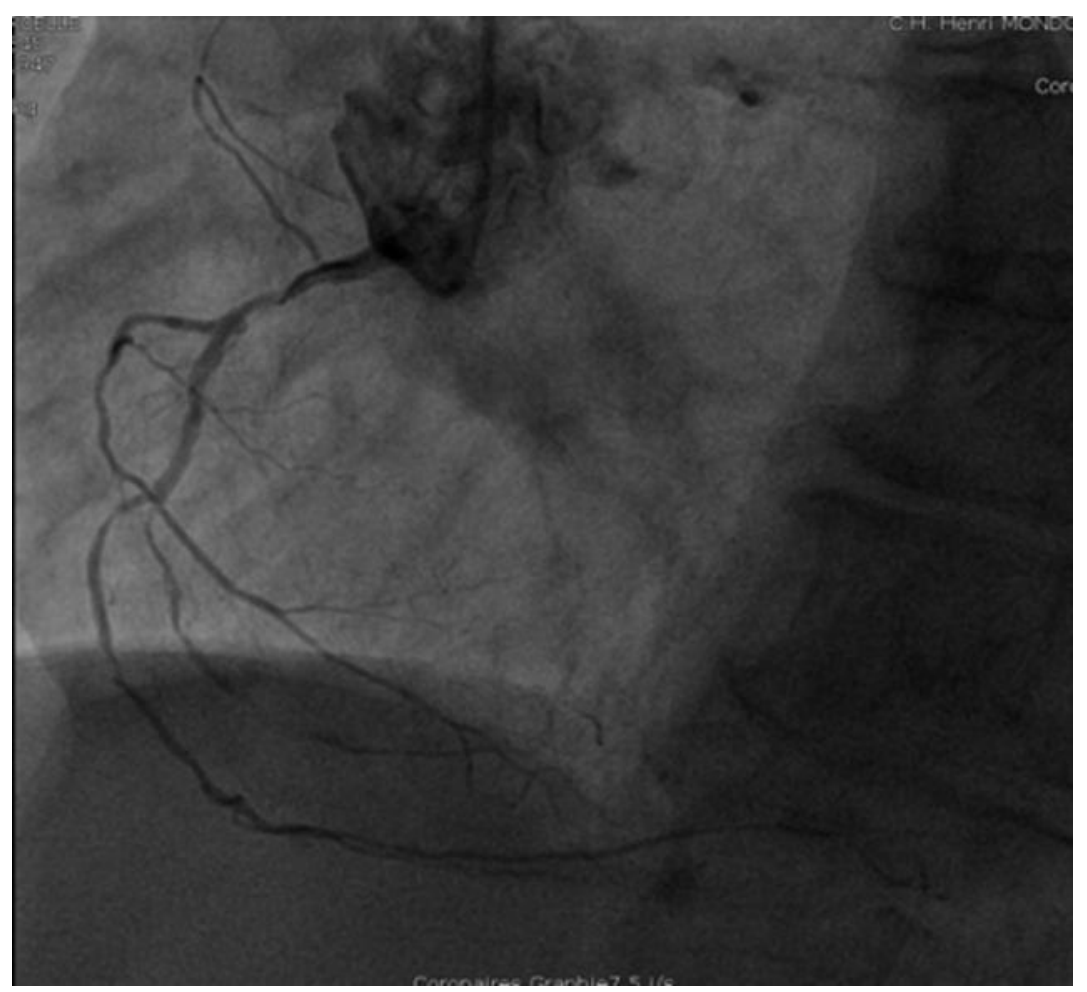

Figure 9:- $30^{\circ} \mathrm{OAG}$ incidence. showing a tight stenosis of the proximal right coronary artery and chronic occlusion of its middle portion. 


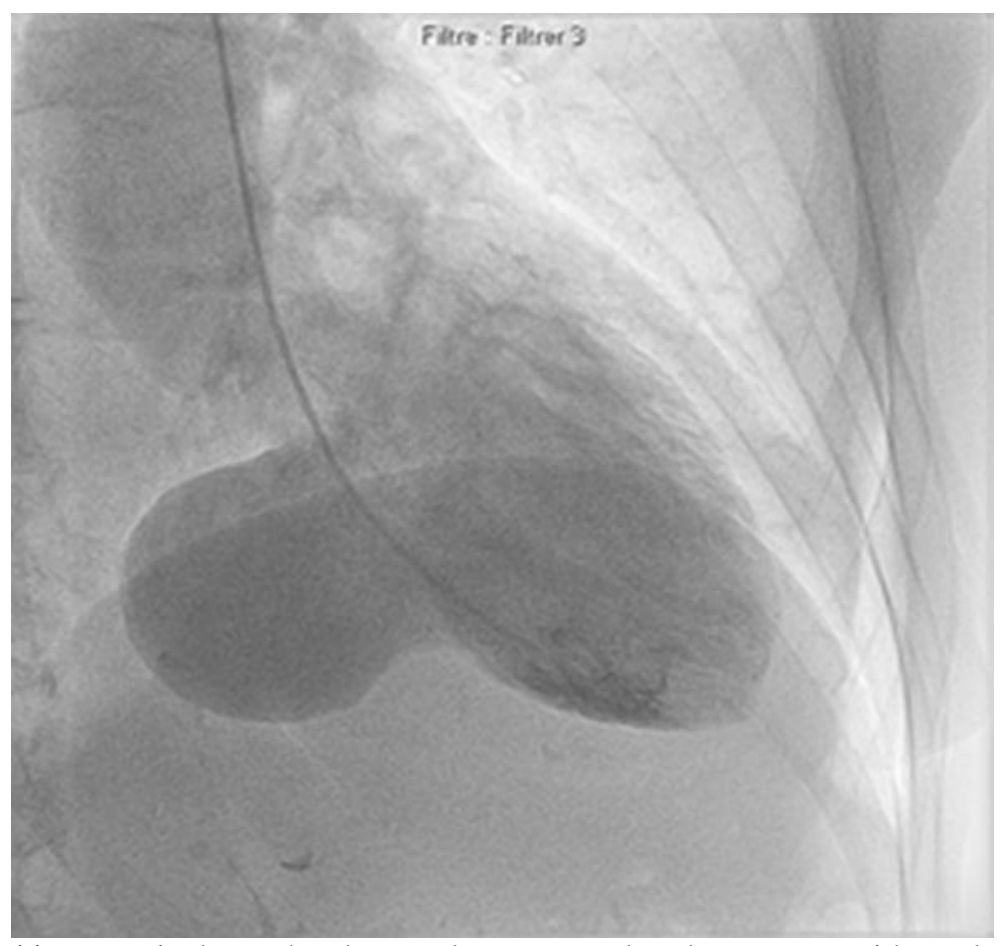

Figure 11:- Ventriculography shows a large poster-basal aneurysm without thrombus.

The patient was transferred to the Clermont Ferrand University Hospital where she underwent heavy cardiac surgery with a triple surgical revascularization (saphenous bisector, right ATI connected in Y on the left breast bridge towards the circumflex lateral, left pedicled breast - IVA 2) associated with a triple Mitro-aortotricuspid valve replacement by bio prosthesis (St Jude $31 \mathrm{~mm}$ mitral and tricuspid and Carpentier Edwards $19 \mathrm{~mm}$ in aortic), LV aneurysm treatment with placement of a hemapatch, resection of tricuspid tumors. Implantation of a SOURIN double-chamber pacemaker in suites (full AVB after surgery).

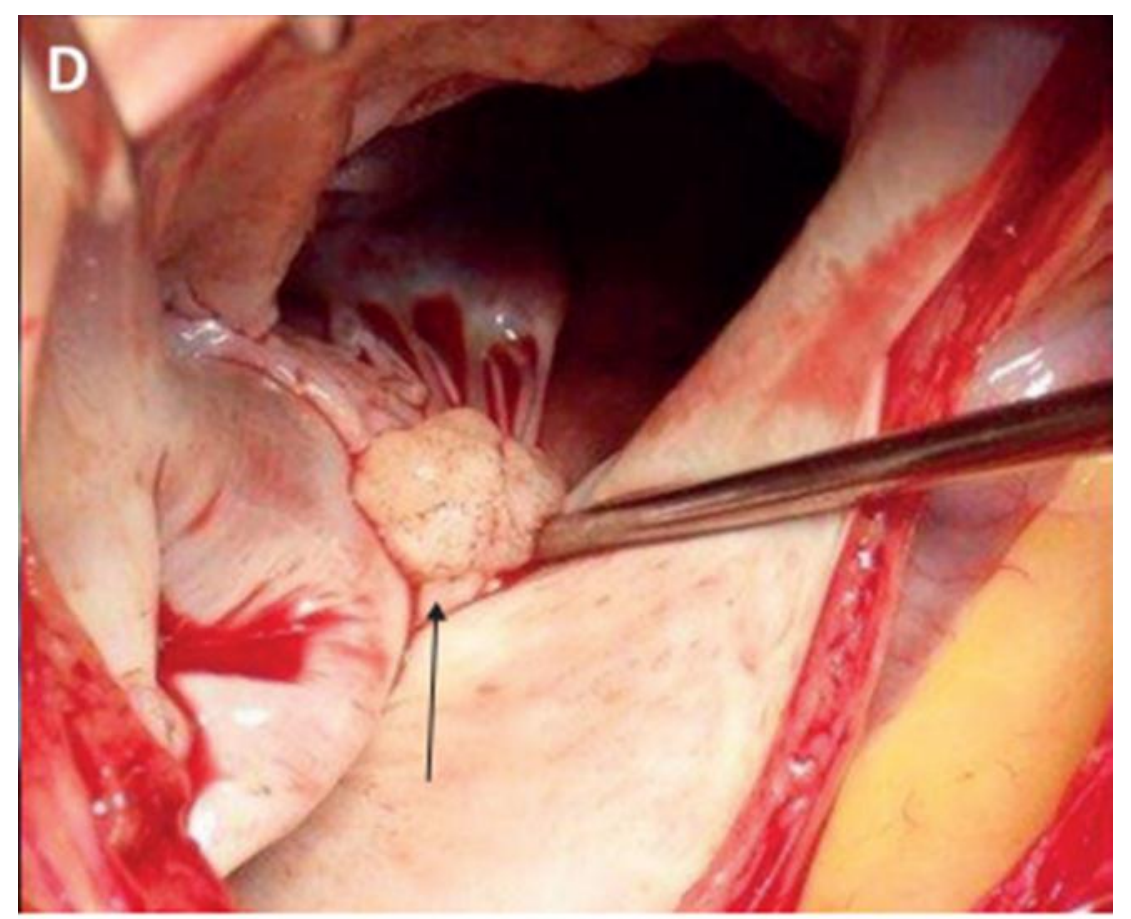

Figure 12:- An operating room showing the fibroelastoma (arrow) close to the external commission. 
The pathology results are in favor of an elastofibroma of the tricuspid valve wall.

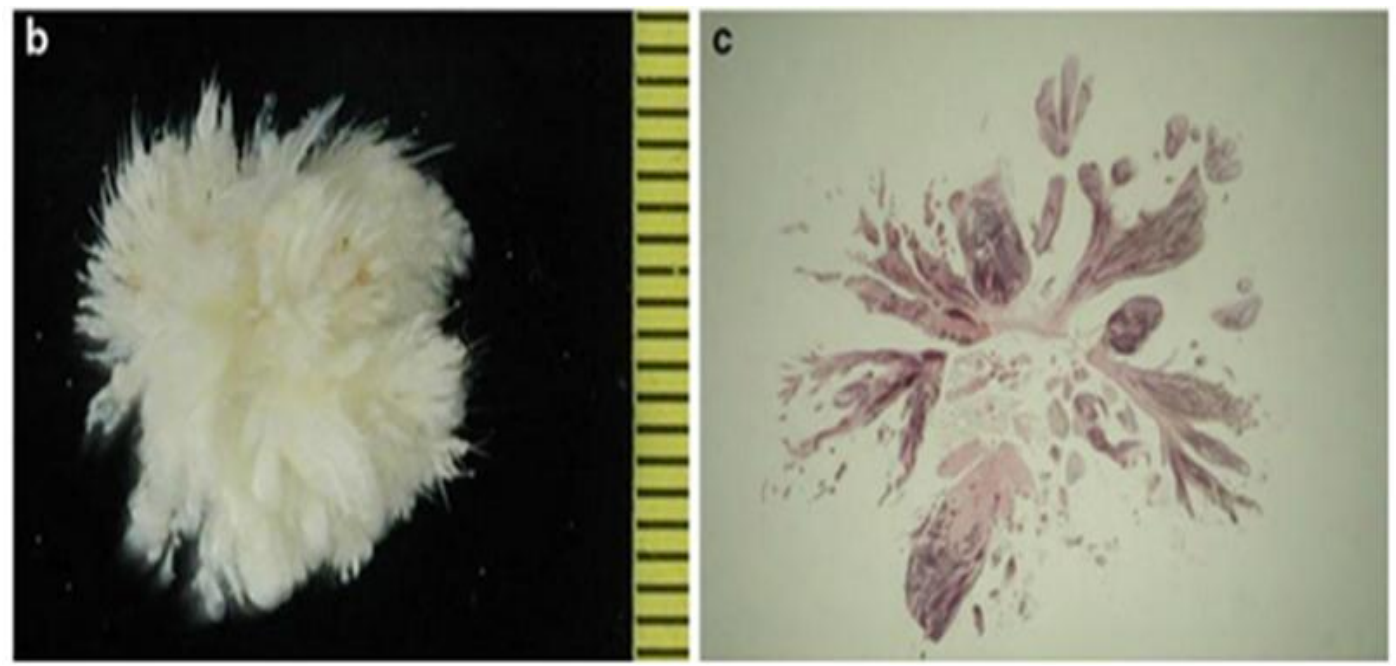

Figure 13:- macroscopic (b) and microscopic (c) aspect of fibroelastoma.

\section{Discussion:-}

Primary heart tumors have an incidence of $0.0017 \%$ to $0.33 \%$. About $75 \%$ of these tumors are benign. Historically, myxomas are the most common and EF is the second benign tumor of the heart (1). However, a Mayo Clinic study published in 2015 found that EF was the most common primary heart tumor (6).

Macroscopically, it resembles a sea Anemone, and there is sometimes an organized thrombus on the surface of the tumor. In $90 \%$ of cases, the tumor is localized at the valve level, most often on the left side and in particular on the aortic valve. More rarely, the tumor is attached to the tricuspid or pulmonary valves or to nonvalvular structures (coronary sinus, inter ventricular or inter atrial septum, ropes, papillary muscles, atrial endocardium, aortic root, right ventricle flushing chamber) [2-4 ]. Unlike vegetation, fibroelastoma is more often inserted into the aortic side of the aortic cusps and the ventricular side of the atrioventricular valves. It is not responsible for any valve dysfunction (3). Cardiac ultrasound, especially transesophageal, has good sensitivity and specificity for diagnosis (88.9\% and $87.8 \%$, respectively) [4]. The tumor has typical ultrasound features:

1. It is round, oval or irregular in shape, often with dotted edges, but well defined, with a homogeneous texture;

2. It is of variable size, between 0,1 and $4 \mathrm{~cm}$, but most often less than $1 \mathrm{~cm}$;

3. It is pedunculated and mobile;

4. It is unique in $90 \%$ of cases [4].

EF usually occurs on the valve endocardium on the left side of the heart. In a retrospective case study of Anastacio et al. (3), out of 23 patients diagnosed with cardiac EF between 1996 and 2012 at the University of Washington, tumors were discovered mainly on the heart valves (aortic, 52\%; mitral, 33\%; tricuspid, 4\%). Only 7\% of patients had origins on the endocardial surface of the ventricular and atrial walls.

The majority of these lesions are solitary and multiple EFs occur only in $7 \%$ to $10 \%$ of patients. In the case studies of Anastacio et al. (7), $82 \%$ of the lesions were completely solitary, $8 \%$ had two separate masses and $8 \%$ had three separate masses, to date, the greatest number of EFs discovered in a single patient was 35 to 40 , found in the two ventricles in a case report published in 2009. (8)

The mass in the right atrium was different from the other masses in that it looked like benign splenic tissue under the light microscope. There have been few cases describing ectopic spleen tissue in the heart. Kuijer et al. (9) published a case report of right atrial and ventricular masses representing splenic tissue. When comparing the heart tumor and the spleen tissue during necropsy, no difference was observed in terms of histological markers. Our case is unique in that our stained for CD8, and the results were negative. Typically, the spleen tissue is CD8 positive. 
EFs are generally asymptomatic and are accidentally identified. In the case studies of Anastacio et al. 313 patients $(57 \%)$ were symptomatic, the most frequent presentation being a thromboembolic event $(33 \%$ stroke and $4 \%$ pulmonary embolism). About $43 \%$ had heart symptoms, including angina, unstable angina or myocardial infarction. Our patient had signs of heart failure + malaise.

The two predictive factors for embolic accidents found in the literature are the size (>10 mm) and the mobility of the tumor $[2,4]$. Surgery is recommended in the case of symptomatic fibroelastoma or mobile asymptomatic tumor, especially if its size is greater than $10 \mathrm{~mm}[1,2]$. For isolated tumors located on the right valves, excision is also indicated in the case of a permeable oval foramen with a consequent right left shunt (4). In symptomatic patients who are not candidates for surgery (for example, patients with high operative risk), long-term anticoagulation may be offered, although we do not have.

No randomized studies that have demonstrated its effectiveness $(2,4)$. In the other cases (asymptomatic patient, small tumor, etc.), medical care is poorly codified. The localization of the mass is the determining factor for the choice of surgical technique (1), but isolated excision of fibroelastoma, without the need for valve replacement, is possible in the majority of aortic and mitral lesions $(3,4)$. Complete surgical resection of fibroelastoma is curative, and the long-term post-operative prognosis is excellent, with no recurrence of the lesions (1-4).

\section{Conclusion:-}

Papillary fibroelastoma is a rare cause of embolic stroke. It is most frequently located in the aortic and mitral valves. The onset of symptoms and tumors that are mobile or larger than $10 \mathrm{~mm}$ are a surgical indication. Complete surgical resection of the tumor is curative, with no recurrence of long-term lesions.

\section{Bibliography:-}

1. Moustafa S, Sauvé C, Pagé P, Serri K. Incidental finding of a papillary fibroelastoma of the mitral valve chordae. Eur J Echocardiogr 2008; 9 (5): 745-6.

2. Gowda RM, Khan IA, Nair CK, Mehta NJ, Vasavada BC, Sacchi TJ. Cardiac papillary fibroelastoma: a comprehensive analysis of 725 cases. Am Heart J 2003; 146 (3): 404-10.

3. Klarich KW, Enriquez-Sarano M, Gura GM, Edwards WD, Tajik AJ, Seward JB. Papillary fibroelastoma: echocardiographic characteristics for diagnosis and pathologic correlation. J Am Coll Cardiol 1997; 30 (3): $784-$ 90.

4. Sun JP, Asher CR, Yang XS et al. Clinical and echocardiographic characteristics of papillary fibroelastomas: a retrospective and prospective study in 162 patients. Circulation 2001; 103 (22): 2687-93.

5. A.O. Petris, D.M. Alexandrescu, Costache II Cardiac tumors

6. Rev Med Chir Soc Med Nat Iasi, 118 (2014), pp. 289-292

7. S.S. Tamin, J.J. Maleszewski, C.G. Scott, S.K. Khan, W.D. Edwards, C.J. Bruce, et al. Prognostic and bioepidemiologic implications of papillary fibroelastomas J Am Coll Cardiol, 65 (2015), pp. 2420-2429

8. $\mathrm{m}$ x m. Anastacio, M.R. Moon, R.J. Damiano Jr., M.K. Pasque, H.S. Maniar, J.S. Lawton Surgical experience with cardiac papillary fibroelastoma over a 15 -year period

9. T.K. Kumar, K. Kuehl, C. Reyes, S. Talwar, A. Moulick, R.A. Jonas Multiple papillary fibroelastomas of the heart Ann Thorac Surg, 88 (2009), pp. e66-e67

10. S. M-9. Said, H.C. Ehlers, S. Hauptmann, S. Hofmann, R. Prondzinsky Papillary fibroelastoma of the aorta Arch Cardiovasc Dis, 101 (2008), pp. 805-806. 